\title{
ON THE INTERSECTION OF TWO PLANE CURVES
}

\author{
Xi Chen
}

\section{Introduction and statement of results}

The following question was raised and partially answered by Geng $\mathrm{Xu}$ in $[\mathrm{X}]$.

Question 1.1. Let $D$ be a general degree $d$ curve in $\mathbb{P}^{2}$. What is the minimal number $i(d, m)$ of points in the set-theoretical intersection $C \cap D$ for any degree $m$ irreducible curve $C$ (suppose that $C$ and $D$ meet properly)?

This problem is related to a conjecture of Kobayashi and Zaidenberg which states that for a sufficiently general curve $D \subset \mathbb{P}^{2}$ of degree $d \geq 5$, as general in the sense that $D$ lies in $\left|\mathcal{O}_{\mathbb{P}^{2}}(d)\right| \cong \mathbb{P}^{d(d+3) / 2}$ with countably many closed proper subvarieties removed, the affine variety $\mathbb{P}^{2} \backslash D$ is hyperbolic. One necessary condition for $\mathbb{P}^{2} \backslash D$ being hyperbolic is that there is no rational curve $C \subset \mathbb{P}^{2}$ meeting $D$ set-theoretically at fewer than three points; otherwise, there is going to be a nonconstant holomorphic map $\mathbb{C} \rightarrow C \backslash(C \cap D) \subset \mathbb{P}^{2} \backslash D$. This property of $\mathbb{P}^{2} \backslash D$ was called "algebraic hyperbolic" in [DSW].

Definition 1.1. A quasi-projective variety is called algebraic hyperbolic if it does not contain a curve whose normalization is an elliptic curve or a rational curve with two points removed, i.e., $\mathbb{P}^{1} \backslash\{p, q\} \cong \mathbb{C}^{*} \cong \operatorname{Spec} \mathbb{C}\left[x, x^{-1}\right]$.

Obviously, hyperbolicity implies algebraic hyperbolicity for smooth quasiprojective varieties.

Using an elegant deformation-theoretical argument, $\mathrm{Xu}$ proved the following [X, Theorem 1].

Theorem $1.1(\mathrm{Xu})$. For $d \geq 3, \min _{m>0} i(d, m)=d-2$.

He thus concluded that every curve $C \subset \mathbb{P}^{2}$ meets $D$ at no less than three distinct points and hence $\mathbb{P}^{2} \backslash D$ is algebraic hyperbolic for a sufficiently general curve $D$ of degree $d \geq 5$. This bound is sharp for $m=1$ and it is achieved by a bitangent or flex line to $D$.

The purpose of this paper is two-fold. First, we will try to sharpen his bound with both $d$ and $m$ fixed. Second, we will try to extend his result to other surfaces.

By dimension count, one may expect that $i(d, m)=d m-r_{d, m}$ where $r_{d, m}$ is the dimension of the linear series cut out on $D$ by all curves of degree $m$, namely,

Received March 13, 2000. 
$r_{d, m}=m(m+3) / 2$ for $m<d$ and $r_{d, m}=d m-(d-1)(d-2) / 2$ for $m \geq d$. However, this is simply false for $m \geq d \geq 3$ by the following construction.

Let $L$ be a bitangent (or flex) line to $D$. Since $D$ is general, $L$ meets $D$ at $d-2$ distinct points. Let $L(X, Y, Z)$ and $D(X, Y, Z)$ be the homogeneous defining equations of $L$ and $D$, respectively. Then for any degree $m-d$ homogeneous polynomial $G(X, Y, Z), L^{m}(X, Y, Z)+D(X, Y, Z) G(X, Y, Z)=0$ defines a degree $m$ curve $C$ which meets $D$ at $d-2$ points, which are the intersections between $L$ and $D$. If we choose $G(X, Y, Z)$ general enough, $C$ is irreducible and actually smooth. Hence, by Xu's result, $i(d, m)=d-2$ for $m \geq d \geq 3$.

Nevertheless, we think that $i(d, m)$ has the expected value for $d>m$, i.e.,

Conjecture 1.1. For $d>m$ and $d \geq 3, i(d, m)=d m-r_{d, m}$.

Although we cannot prove the above conjecture, we have the following estimate for $i(d, m)$ when $m<d$.

Theorem 1.2. For $d>m$,

$$
i(d, m) \geq \min \left(d m-\frac{m(m+3)}{2}, 2 d m-2 m^{2}-2\right) .
$$

An easy corollary of the above theorem is the following

Corollary 1.1. For $2 d \geq 3 m-2$ and $d \geq 3, i(d, m)=d m-m(m+3) / 2$, i.e., Conjecture 1.1 holds for $2 d \geq 3 m-2$. In particular, it holds for $m \leq 4$.

In order to formulate Kobayashi type conjectures on surfaces other than $\mathbb{P}^{2}$, we need to study Question 1.1 in the following general setting.

Question 1.2. Let $S$ be a smooth surface and let $L$ and $M$ be two line bundles on $S$. Let $D$ be a general member of $|L|$. What is the minimal number $i(L, M)$ of points in the set-theoretical intersection $C \cap D$ for any irreducible curve $C \in|M|$ (suppose that $C$ and $D$ meet properly)?

We will work on rational ruled surfaces, although our method can be extended to other surfaces. By convention, let $\mathbb{F}_{n}$ be the rational ruled surface given by $\mathbb{P}\left(\mathcal{O}_{\mathbb{P}^{1}} \oplus \mathcal{O}_{\mathbb{P}^{1}}(n)\right)$ over $\mathbb{P}^{1}$ and let $C$ and $F$ be the zero section and the fiber of $\mathbb{F}_{n} \rightarrow \mathbb{P}^{1}$, i.e., $C^{2}=-n, C \cdot F=1$ and $F^{2}=0$. We have the following lower bound for $i(L, M)$ with $L$ ample.

Theorem 1.3. Let $L=\mathcal{O}(a C+b F)$ be an ample line bundle on $\mathbb{F}_{n}$ with $a \geq 2$ and $b \geq 2$. Then $\min _{M} i(L, M)=\min (a-1, b-a n, b-n-1)$, where $M$ runs over all line bundles with irreducible general global sections.

It follows immediately from Theorem 1.3 that every curve on $\mathbb{F}_{n}$ meets $D$ at no less than three distinct points for a sufficiently general $D \in|a C+b F|$ with $a \geq 4$ and $b \geq \max (4+n, 3+a n)$. Therefore

Corollary 1.2. For a sufficiently general curve $D \in|a C+b F|$ on $\mathbb{F}_{n}$ with $a \geq 4$ and $b \geq \max (4+n, 3+$ an $)$, the complement $\mathbb{F}_{n} \backslash D$ is algebraic hyperbolic. 
Notice that the bound in Theorem 1.3 can be achieved by a curve in $|C|$ or $|F|$, which is necessarily a rational curve. So the lower bounds for $a$ and $b$ in the above corollary cannot be improved.

This enables us to formulate Kobayashi conjecture on $\mathbb{F}_{n}$.

Conjecture 1.2 (Kobayashi Conjecture on Rational Ruled Surfaces). For a sufficiently general curve $D \in|a C+b F|$ on $\mathbb{F}_{n}$ with $a \geq 4$ and $b \geq \max (4+$ $n, 3+$ an), the complement $\mathbb{F}_{n} \backslash D$ is hyperbolic.

The organization of this paper is as follows. Theorem 1.2 and 1.3 will be proved in the next two sections, respectively. At the end of the third section, we will also discuss some related problems.

Throughout this paper we work exclusively over the field of complex numbers $\mathbb{C}$.

\section{Proof of Theorem 1.2}

Let $W_{\delta} \subset|\mathcal{O}(m)| \times|\mathcal{O}(d)|$ be the incidence correspondence defined by

$$
\begin{aligned}
W_{\delta}=\{(C, D): C \in|\mathcal{O}(m)| \text { is irreducible, } D \in|\mathcal{O}(d)| \text { is smooth, } \\
\\
\text { and } C \text { and } D \text { meet set-theoretically at } \delta \text { points }\}
\end{aligned}
$$

Our proof of Theorem 1.2 is carried out by estimating the dimension of $W_{\delta}$ and show that $\operatorname{dim} W_{\delta}<\operatorname{dim}|\mathcal{O}(d)|$ if $\delta<d m-m(m+3) / 2$ and $\delta<2 d m-2 m^{2}-2$ and hence it cannot dominate $|\mathcal{O}(d)|$ in this case.

Let $\pi: W_{\delta} \rightarrow|\mathcal{O}(m)|$ be the projection of $W_{\delta}$ to $|\mathcal{O}(m)|$ and let $C$ be a general point of $\pi\left(W_{\delta}\right)$ (by a general point, we mean a general point of an irreducible component of $\left.\pi\left(W_{\delta}\right)\right)$.

Let $\pi_{C}$ be the fiber of $\pi: W_{\delta} \rightarrow|\mathcal{O}(m)|$ over $C$ and let $(C, D)$ be a general point on $\pi(C)$. There exists a series of blowups of $\mathbb{P}^{2}$ such that the proper transforms $\widetilde{C}$ and $\widetilde{D}$ of $C$ and $D$ meet at smooth points on both curves (since we assume that $D$ is smooth, we only have to resolve the singularities of $C$ where $D$ passes through). Let $\widetilde{\mathbb{P}^{2}}$ be the resulting blowup of $\mathbb{P}^{2}$ and $E_{i}(1 \leq i \leq \alpha)$ be the exceptional divisors. Suppose that $\widetilde{C} \in \mid \mathcal{O}\left(m H-\sum_{i=1}^{\alpha} r_{i} E_{i}\right)$ and $\widetilde{D} \in$ $\left|\mathcal{O}\left(d H-\sum_{i=1}^{\alpha} E_{i}\right)\right|$, where $r_{i}>1$ and $H$ is the pull-back of the hyperplane divisor of $\mathbb{P}^{2}$.

Suppose that $\widetilde{C}$ and $\widetilde{D}$ meet at points $p_{1}, p_{2}, \ldots, p_{\beta}$ with multiplicities $m_{1}, m_{2}, \ldots, m_{\beta}$, respectively, where $\beta \leq \delta \leq \alpha+\beta$. Then by a deformationtheoretical argument, the tangent space $T_{\pi_{C},(C, D)}$ of $\pi_{C}$ at $(C, D)$ is contained in

$$
\begin{aligned}
& H^{0}\left(\mathcal{O}_{D}\left(d H-\sum_{i=1}^{\alpha} E_{i}\right) \otimes \mathcal{O}_{D}\left(-\sum_{j=1}^{\beta}\left(m_{j}-1\right) p_{j}\right)\right) \\
= & H^{0}\left(\mathcal{O}_{\tilde{D}}\left((d-m) H+\sum_{i=1}^{\alpha}\left(r_{i}-1\right) E_{i}\right) \otimes \mathcal{O}_{D}\left(\sum_{j=1}^{\beta} p_{j}\right)\right) .
\end{aligned}
$$

Hence by Riemann-Roch 


$$
\begin{aligned}
\operatorname{dim} \pi_{C} & \leq h^{0}\left(\mathcal{O}_{D}\left((d-m) H+\sum_{i=1}^{\alpha}\left(r_{i}-1\right) E_{i}+\sum_{j=1}^{\beta} p_{j}\right)\right) \\
& =\frac{d(d+3)}{2}+\sum_{i=1}^{\alpha}\left(r_{i}-1\right)+\beta-d m \\
& +h^{0}\left(\mathcal{O}_{D}\left((m-3) H-\sum_{i=1}^{\alpha}\left(r_{i}-1\right) E_{i}\right) \otimes \mathcal{O}_{D}\left(-\sum_{j=1}^{\beta} p_{j}\right)\right) .
\end{aligned}
$$

It is not hard to see that

$$
\begin{aligned}
& h^{0}\left(\mathcal{O}_{D}\left((m-3) H-\sum_{i=1}^{\alpha}\left(r_{i}-1\right) E_{i}\right) \otimes \mathcal{O}_{D}\left(-\sum_{j=1}^{\beta} p_{j}\right)\right) \\
\leq & h^{0}\left(\mathcal{O}_{C}\left((m-3) H-\sum_{i=1}^{\alpha}\left(r_{i}-1\right) E_{i}\right) \otimes \mathcal{O}_{C}\left(-\sum_{j=1}^{\beta} p_{j}\right)\right) \\
& +\sum_{i=1}^{\alpha} \frac{\left(r_{i}-1\right)\left(r_{i}-2\right)}{2}
\end{aligned}
$$

This can be shown by the following argument.

We further blow up $\widetilde{\mathbb{P}^{2}}$ at points $p_{1}, p_{2}, \ldots, p_{\beta}$ with corresponding exceptional divisors $F_{1}, F_{2}, \ldots, F_{\beta}$. We still denote the resulting surface by $\widetilde{\mathbb{P}^{2}}$ and the proper transforms of $C$ and $D$ by $\widetilde{C}$ and $\widetilde{D}$. We have the following exact sequence on $\widetilde{\mathbb{P}^{2}}$

$$
\begin{aligned}
0 & \rightarrow H^{0}\left((m-d-3) H-\sum_{i=1}^{\alpha}\left(r_{i}-2\right) E_{i}\right) \\
& \rightarrow H^{0}\left((m-3) H-\sum_{i=1}^{\alpha}\left(r_{i}-1\right) E_{i}-\sum_{j=1}^{\beta} F_{j}\right) \\
& \rightarrow H^{0}\left(\mathcal{O}_{D}\left((m-3) H-\sum_{i=1}^{\alpha}\left(r_{i}-1\right) E_{i}-\sum_{j=1}^{\beta} F_{j}\right)\right) \\
& \rightarrow H^{1}\left((m-d-3) H-\sum_{i=1}^{\alpha}\left(r_{i}-2\right) E_{i}\right) .
\end{aligned}
$$

Obviously, $h^{0}\left((m-d-3) H-\sum_{i=1}^{\alpha}\left(r_{i}-2\right) E_{i}\right)=0$ and

$$
\begin{aligned}
& h^{2}\left((m-d-3) H-\sum_{i=1}^{\alpha}\left(r_{i}-2\right) E_{i}\right) \\
= & h^{0}\left((d-m) H+\sum_{i=1}^{\alpha}\left(r_{i}-1\right) E_{i}+\sum_{j=1}^{\beta} F_{j}\right) \\
= & \frac{(d-m)(d-m+3)}{2}+1 .
\end{aligned}
$$


Hence by Riemann-Roch,

$$
h^{1}\left((m-d-3) H-\sum_{i=1}^{\alpha}\left(r_{i}-2\right) E_{i}\right)=\sum_{i=1}^{\alpha} \frac{\left(r_{i}-1\right)\left(r_{i}-2\right)}{2} .
$$

Therefore

$$
\begin{aligned}
& h^{0}\left(\mathcal{O}_{D}\left((m-3) H-\sum_{i=1}^{\alpha}\left(r_{i}-1\right) E_{i}-\sum_{j=1}^{\beta} F_{j}\right)\right) \\
\leq & h^{0}\left((m-3) H-\sum_{i=1}^{\alpha}\left(r_{i}-1\right) E_{i}-\sum_{j=1}^{\beta} F_{j}\right)+\sum_{i=1}^{\alpha} \frac{\left(r_{i}-1\right)\left(r_{i}-2\right)}{2} .
\end{aligned}
$$

Similarly, we have

$$
\begin{aligned}
& h^{0}\left(\mathcal{O}_{C}\left((m-3) H-\sum_{i=1}^{\alpha}\left(r_{i}-1\right) E_{i}-\sum_{j=1}^{\beta} F_{j}\right)\right) \\
= & h^{0}\left((m-3) H-\sum_{i=1}^{\alpha}\left(r_{i}-1\right) E_{i}-\sum_{j=1}^{\beta} F_{j}\right) .
\end{aligned}
$$

Combining (2.2) and (2.3), we obtain (2.1). Therefore

$$
\begin{aligned}
\operatorname{dim} \pi_{C} & \leq \frac{d(d+3)}{2}+\sum_{i=1}^{\alpha} \frac{r_{i}\left(r_{i}-1\right)}{2}+\beta-d m \\
& +h^{0}\left(\mathcal{O}_{C}\left((m-3) H-\sum_{i=1}^{\alpha}\left(r_{i}-1\right) E_{i}\right) \otimes \mathcal{O}_{C}\left(-\sum_{j=1}^{\beta} p_{j}\right)\right) \\
& =\frac{d(d+3)}{2}+\sum_{i=1}^{\alpha} \frac{r_{i}\left(r_{i}-1\right)}{2}+\beta-d m+h^{0}\left(\omega_{C} \otimes \mathcal{O}_{C}\left(-\sum_{j=1}^{\beta} p_{j}\right)\right)
\end{aligned}
$$

where $\omega_{C}$ is the dualizing sheaf of $\widetilde{C}$.

By Clifford's theorem (see for example [ACGH, pp. 107-8]), we have either

$$
h^{0}\left(\omega_{C} \otimes \mathcal{O}_{C}\left(-\sum_{j=1}^{\beta} p_{j}\right)\right)=0
$$

or

$$
h^{0}\left(\mathcal{O}_{C}\left(\sum_{j=1}^{\beta} p_{j}\right)\right) \leq \beta / 2+1 .
$$

Hence correspondingly, we have either

$$
\operatorname{dim} \pi_{C} \leq \frac{d(d+3)}{2}+\sum_{i=1}^{\alpha} \frac{r_{i}\left(r_{i}-1\right)}{2}+\beta-d m
$$


or

$$
\operatorname{dim} \pi_{C} \leq \frac{d(d+3)}{2}+\frac{\beta}{2}-d m+\frac{(m-1)(m-2)}{2} .
$$

Since $C$ is a general member of $\pi\left(W_{\delta}\right)$ and $C$ has singularities with multiplicities $r_{i}(1 \leq i \leq \alpha)$, by Zariski's theorem on the deformation of planary curve singularities $[\mathrm{Z}]$, we have

$$
\operatorname{dim} \pi\left(W_{\delta}\right) \leq \frac{m(m+3)}{2}-\sum_{i=1}^{\alpha} \frac{r_{i}\left(r_{i}-1\right)}{2} .
$$

And hence we have either

$$
\operatorname{dim} W_{\delta} \leq \frac{d(d+3)}{2}+\beta-d m+\frac{m(m+3)}{2}
$$

or

$$
\operatorname{dim} W_{\delta} \leq \frac{d(d+3)}{2}+\frac{\beta}{2}-d m+m^{2}+1-\sum_{i=1}^{\alpha} \frac{r_{i}\left(r_{i}-1\right)}{2} .
$$

Therefore, if $W_{\delta}$ dominates $|\mathcal{O}(d)|$, we necessarily have

$$
\delta \geq \beta \geq \min \left(d m-\frac{m(m+1)}{2}, 2\left(d m-m^{2}-1\right)\right) .
$$

This finishes the proof of Theorem 1.2.

\section{Intersections of Two Curves on Rational Ruled Surfaces}

Our approach to Theorem 1.3 is different from that of Xu's. A key ingredient of Xu's proof of Theorem 1.1 is a map from the deformation space of the pair $(D, E)$, where $D \in\left|\mathcal{O}_{\mathbb{P}^{2}}(d)\right|$ and $E \in\left|\mathcal{O}_{\mathbb{P}^{2}}(m)\right|$ meet at no less than $s$ distinct points, to the cohomology group of a sheaf over $D$. More specifically, let $\left(Z_{0}, Z_{1}, Z_{2}\right)$ be generic homogeneous coordinates of $\mathbb{P}^{2}$ and let $F_{0} \in H^{0}\left(\mathcal{O}_{\mathbb{P}^{2}}(d)\right)$ and $G_{0} \in H^{0}\left(\mathcal{O}_{\mathbb{P}^{2}}(m)\right)$ be the defining equations of $D$ and $E$. A first order deformation of $(D, E)$ is given by $F_{1} \in H^{0}\left(\mathcal{O}_{\mathbb{P}^{2}}(d)\right)$ and $G_{1} \in H^{0}\left(\mathcal{O}_{\mathbb{P}^{2}}(m)\right)$ such that the curves $\left\{F_{0}+t F_{1}=0\right\}$ and $\left\{G_{0}+t G_{1}=0\right\}$ meet at no less than $s$ points over the ring $\mathbb{C}[t] /\left(t^{2}\right)$. It is observed by $\mathrm{Xu}$ that $[\mathrm{X}$, Lemma 1$]$

$$
\frac{\partial F_{0}}{\partial Z_{i}} G_{1}-\frac{\partial G_{0}}{\partial Z_{i}} F_{1} \in H^{0}\left(D, \mathcal{O}_{D}(d+m-1) \otimes \mathcal{O}_{D}\left(-\sum_{j=1}^{s}\left(\mu_{j}-1\right) p_{j}\right)\right)
$$

for $i=0,1,2$, where $D$ and $E$ meet at $p_{1}, p_{2}, \ldots, p_{s}$ with multiplicities $\mu_{1}, \mu_{2}, \ldots, \mu_{s}$, respectively.

The relation (3.1) forms the basis of Xu's proof of Theorem 1.1. If we were to prove Theorem 1.3 following Xu's line of argument, we would have to come up with a relation similar to $(3.1)$ on $\mathbb{F}_{n}$, which we are unable to do. So we find that $\mathrm{Xu}$ 's analysis, though ingenious on its own, is hard, if not impossible, to carry out on surfaces other than $\mathbb{P}^{2}$. Therefore, we will adopt a different approach to Theorem 1.3, which is based upon degeneration and induction. 
Let $\Delta$ be a disk parameterized by $t$ and let $Y \subset \mathbb{F}_{n} \times \Delta$ be a pencil of curves in $|a C+b F|$ whose central fiber $Y_{0}=G \cup \Gamma$ is reducible with two components $G \in|(a-1) C+(b-n-1) F|$ and $\Gamma \cong \mathbb{P}^{1} \in|C+(n+1) F|$. Let $X \subset \mathbb{F}_{n} \times \Delta$ be a family of curves on $\mathbb{F}_{n}$ whose general fiber $X_{t}(t \neq 0)$ meets $Y_{t}$ at $s$ distinct points (a base change may be needed to ensure the existence of $X$ ). If $X_{0}$ meets $Y_{0}$ properly, we may deduce $s \geq \min (a-1, b-a n, b-n-1)$ by the induction hypothesis that

$$
\begin{aligned}
\#\left(X_{0} \cap G\right) & \geq \min (a-2, b-a n-1, b-n-2) \\
& =\min (a-1, b-a n, b-n-1)-1
\end{aligned}
$$

and by the fact that $\#\left(X_{0} \cap \Gamma\right) \geq 1$, where we use the notation $\#(A \cap B)$ to denote the number of points in the set-theoretical intersection $A \cap B$ between the two curves $A$ and $B$. Of course, some care has to be taken to make sure that $X_{0}$ meets $\Gamma$ at at least one point outside of $G \cap \Gamma$ (see below). Unfortunately, $X_{0}$ may very well contain $G$ or $\Gamma$ as a component. So we have to regard $\left|\mathcal{O}_{Y_{0}}\left(X_{0}\right)\right|$ as the limit linear series $\lim _{t \rightarrow 0}\left|\mathcal{O}_{Y_{t}}\left(X_{t}\right)\right|$ and, correspondingly, $Y_{0} \cap X_{0}$ as the limit of the section $Y_{t} \cap X_{t}$ in $\left|\mathcal{O}_{Y_{t}}\left(X_{t}\right)\right|$. For an introduction to the theory of limit linear series, please see, for example, [E-H] or [H, Chap. 5].

For the purpose of induction, we will prove Theorem 1.3 in the following slightly more general form.

Proposition 3.1. Let $L=\mathcal{O}(a C+b F)$ be an ample line bundle on $\mathbb{F}_{n}$ with $a \geq 2$ and $b \geq 2$. Then for a sufficiently general curve $D \in|L|$,

1. $\#(D \cap E) \geq \min (a-1, b-a n, b-n-1)$ for any curve $E \subset \mathbb{F}_{n}$ that meets $D$ properly;

2. in addition, there exists a set $\Sigma_{D}$ consisting of countably many points on $D$ such that if $\#(D \cap E)=\min (a-1, b-a n, b-n-1)$ for some $E$, $(D \cap E) \subset \Sigma$.

We prove Proposition 3.1 by induction on $\min (a-1, b-a n, b-n-1)$.

If $\min (a-1, b-a n, b-n-1)=1$, we only need to verify the second part of the proposition. Notice that $D$ has genus $g(D)=1+\frac{1}{2}(a-2)(b-a n)+\frac{1}{2} a(b-n-2) \geq$ 1. If $D$ meets $E$ at a single $p$ for some $E, \mathcal{O}_{D}(\mu p)=\mathcal{O}_{D}(E)$, where $\mu=D \cdot E$. If we fix the divisor class of $E$, there are only finitely many points $p$ with this property since $g(D) \geq 1$. Therefore, there are only countably many points $p$ such that $D \cap E=\{p\}$ for some $E$.

Suppose that $\min (a-1, b-a n, b-n-1) \geq 2$. Notice that $\mathcal{O}((a-1) C+(b-$ $n-1) F$ ) is ample under this assumption.

Let $X, Y, G$ and $\Gamma$ be defined as before. Suppose that $G$ and $\Gamma$ meet at points $p_{1}, p_{2}, \ldots, p_{l}$, where $l=a+b-n-2$. Let $M=\mathcal{O}\left(X_{t}\right)$ be the line bundle associated to $X_{t}$.

Let $\sigma_{t} \in\left|\mathcal{O}_{Y_{t}}\left(X_{t}\right)\right|$ be the section cut out by $X_{t}$ on $Y_{t}$ and let $\sigma_{0}=\lim _{t \rightarrow 0} \sigma_{t}$. Let $\sigma_{\Gamma}=\left.\sigma_{0}\right|_{\Gamma}$ and $\sigma_{G}=\left.\sigma_{0}\right|_{G}$ be the restrictions of $\sigma_{0}$ to $\Gamma$ and $G$, respectively. Then $\sigma_{\Gamma}$ is a section in

$$
\left|\mathcal{O}_{\Gamma}\left(\mu\left(p_{1}+p_{2}+\ldots+p_{l}\right)\right) \otimes M\right|=\left|\mathcal{O}_{\Gamma}(\mu G) \otimes M\right|
$$


and $\sigma_{G}$ is a section in

$$
\left|\mathcal{O}_{G}\left(-\mu\left(p_{1}+p_{2}+\ldots+p_{l}\right)\right) \otimes M\right|=\left|\mathcal{O}_{G}(-\mu \Gamma) \otimes M\right|
$$

where $\mu$ is an integer and $\sigma_{\Gamma}$ and $\sigma_{G}$ are cut out by sections in $|\mathcal{O}(\mu G) \otimes M|$ and $|\mathcal{O}(-\mu \Gamma) \otimes M|$, respectively.

Suppose that $\mathcal{O}(-\mu \Gamma) \otimes M$ is nontrivial. Then by induction hypothesis $\sigma_{G}$ vanishes at no less than $\min (a-2, b-a n-1, b-n-2)$ distinct points. If $\sigma_{\Gamma}$ vanishes at at least one point other than $p_{1}, p_{2}, \ldots, p_{l}$, we are done; if not, we have either $\mathcal{O}(\mu G) \otimes M$ is trivial and $\sigma_{\Gamma}$ is nowhere vanishing or $\sigma_{\Gamma}$ only vanishes at $p_{1}, p_{2}, \ldots, p_{l}$.

If $\mathcal{O}(\mu G) \otimes M$ is trivial and $\sigma_{\Gamma}$ is nowhere vanishing, then for any two points among $p_{1}, p_{2}, \ldots, p_{l}$, say $p_{1}$ and $p_{2}$, the ratio $\sigma_{G}\left(p_{1}\right) / \sigma_{G}\left(p_{2}\right)$ is uniquely determined by the choice of the pencil $Y$. Actually we have the following very explicit relation

$$
\frac{\sigma_{G}\left(p_{1}\right)}{\sigma_{G}\left(p_{2}\right)}=\left(\frac{f\left(p_{1}\right)}{f\left(p_{2}\right)}\right)^{-\mu}
$$

where $f \in|L|$ is the section which cuts out a general member $Y_{t}$ of the pencil $Y$. If $\sigma_{G}$ vanishes at more than $\min (a-2, b-a n-1, b-n-2)$ distinct points, there is nothing to prove; otherwise, $\sigma_{G}$ vanishes at exactly $\min (a-2, b-a n-1, b-n-2)$ distinct points. Then by induction hypothesis, there are only countably many possible choices of $\sigma_{G}$. However, by (3.2), the ratio $\sigma_{G}\left(p_{1}\right) / \sigma_{G}\left(p_{2}\right)$ can be made into an arbitrary complex value by a choice of $f$ (and thus a choice of the pencil $Y)$. Contradiction.

If $\sigma_{\Gamma}$ only vanishes at $p_{1}, p_{2}, \ldots, p_{l}$, since we have already taken care of the case that $\mathcal{O}(\mu G) \otimes M$ is trivial and $\sigma_{\Gamma}$ is nowhere vanishing, we may assume that $\sigma_{\Gamma}$ vanishes at at least one point among $p_{1}, p_{2}, \ldots, p_{l}$, say $p_{1}$. Then $\sigma_{G}$ must vanish at $p_{1}$ as well. Again, if $\sigma_{G}$ vanishes at more than $\min (a-2, b-a n-1, b-n-2)$ distinct points, there is nothing to prove; otherwise, $\sigma_{G}$ vanishes at exactly $\min (a-2, b-a n-1, b-n-2)$ distinct points. By induction hypothesis, $p_{1} \in \Sigma_{G}$. But if we choose $\Gamma$ generically, $p_{1} \notin \Sigma_{G}$. Contradiction.

Now suppose that $\mathcal{O}(-\mu \Gamma) \otimes M$ is trivial. If $\sigma_{G}=0$, then $\sigma_{\Gamma}$ vanishes at $p_{1}, p_{2}, \ldots, p_{l}$ and $l=a+b-n-2>\min (a-1, b-a n, b-n-1)$; we are done. Otherwise, $\sigma_{G}$ is no where vanishing. The ratio $\sigma_{\Gamma}\left(p_{i}\right) / \sigma_{\Gamma}\left(p_{j}\right)$ for any two points $p_{i}$ and $p_{j}$ among $p_{1}, p_{2}, \ldots, p_{l}$, just as in (3.2), is uniquely determined by the choice of $Y$ and is given by

$$
\frac{\sigma_{\Gamma}\left(p_{i}\right)}{\sigma_{\Gamma}\left(p_{j}\right)}=\left(\frac{f\left(p_{i}\right)}{f\left(p_{j}\right)}\right)^{\mu} .
$$

The rational map $|L| \rightarrow \mathbb{P}^{l-1}$ by sending $f \in|L|$ to

$$
\left(f^{\mu}\left(p_{1}\right), f^{\mu}\left(p_{2}\right), \ldots, f^{\mu}\left(p_{l}\right)\right)
$$

is dominant due to the facts that $H^{0}\left(\mathbb{F}_{n}, L\right)$ surjects onto $H^{0}(\Gamma, L)$ and $L \otimes$ $\mathcal{O}_{\Gamma}\left(-\sum_{i \neq j} p_{i}\right)$ is base point free on $\Gamma$ for each $1 \leq j \leq l$. On the other hand, 
the space

$$
\left\{\sigma_{\Gamma} \mid \sigma_{\Gamma} \text { vanishes at less than } l-1 \text { distinct points }\right\}
$$

has dimension $l-2$ and hence cannot dominate $\mathbb{P}^{l-1}$. So $\sigma_{\Gamma}$ vanishes at at least $l-1>\min (a-1, b-a n, b-n-1)$ distinct points for a general choice of $f$ by $(3.3)$.

This finishes the proof of the first part of the proposition.

Suppose that $\sigma_{0}$ vanishes at exactly $\min (a-1, b-a n, b-n-1)$ distinct points. This can happen only when $\mathcal{O}(-\mu \Gamma) \otimes M$ is nontrivial.

Suppose that $\sigma_{G}$ vanishes at exactly $\min (a-2, b-a n-1, b-n-2)$ distinct points. Our previous argument shows that $\sigma_{G}$ does not vanish at $p_{1}, p_{2}, \ldots, p_{l}$ for a general choice of $G \cup \Gamma$. Then $\sigma_{\Gamma}$ must vanish at a single point $p \notin$ $\left\{p_{1}, p_{2}, \ldots, p_{l}\right\}$. Since $\#(G \cap \Gamma) \geq 2$, the natural map from $Y_{0} \backslash\left\{p_{1}, p_{2}, \ldots, p_{l}\right\}$ to $\operatorname{Pic}\left(Y_{0}\right)$ is injective. So $p$ is determined up to finitely many possibilities by $M$ and the vanishing locus of $\sigma_{G}$. By induction, the vanishing locus of $\sigma_{G}$ is contained in some countable set $\Sigma_{G}$ depending only on $G$. So the vanishing locus of $\sigma_{0}$ is also contained in some countable set $\Sigma_{G \cup \Gamma}$ depending only on $G \cup \Gamma$.

Suppose that $\sigma_{G}$ vanishes at exactly $\min (a-1, b-a n, b-n-1)$ distinct points and suppose that there is a one-parameter family of $\sigma_{0}(u)$ with this property, where $\sigma_{0}(u)$ is parameterized by $u \in U$ for some irreducible curve $U$.

Suppose that $\mathcal{O}(\mu G) \otimes M$ is trivial. There exists $u_{0} \in U$ such that $\sigma_{G}\left(u_{0}\right)$ vanishes at $p_{1}$. Since $\mathcal{O}(\mu G) \otimes M$ is trivial, $\sigma_{\Gamma}\left(u_{0}\right)=0$ and hence $\sigma_{G}\left(u_{0}\right)$ vanishes at $p_{1}, p_{2}, \ldots, p_{l}$. But $l>\min (a-1, b-a n, b-n-1)$. Contradiction.

Suppose that $\mathcal{O}(\mu G) \otimes M$ is nontrivial. Then $\sigma_{\Gamma}(u)$ vanishes at at least one point among $p_{1}, p_{2}, \ldots, p_{l}$, say $p_{1}$. Hence $\sigma_{G}(u)$ vanishes at $p_{1}$ for all $u \in U$. As $u$ varies, another vanishing point of $\sigma_{G}(u)$ will approach $p_{1}$. So there exists $u_{0} \in U$ such that $\sigma_{G}\left(u_{0}\right)$ vanishes at $\min (a-2, b-a n-1, b-n-2)$ distinct points and among them vanishes at a general point $p_{1}$. Again this is impossible by induction. Contradiction.

This finishes the proof of Proposition 3.1.

The degeneration method we used can be applied to surfaces other than rational ruled surfaces. For example, we can give an alternative proof of Xu's Theorem 1.1 by degenerating a degree $d$ curve to a union of a degree $d-1$ curve and a line and arguing by induction.

A proof of Xu's Theorem 1.1 via degeneration. As in the case of Proposition 3.1, we need to add a clause to the theorem for the purpose of induction, i.e., we will prove the following statement by induction on $d$.

For a sufficiently general curve $D$ of degree $d \geq 3$ in $\mathbb{P}^{2}, \#(D \cap E) \geq d-2$ for any curve $E \subset \mathbb{P}^{2}$ that meets $D$ properly. In addition, there exists a set $\Sigma_{D}$ of countably many points on $D$ such that if $\#(D \cap E)=d-2$ for some $E$, $(D \cap E) \subset \Sigma_{D}$.

Let $Y \subset \mathbb{P}^{2} \times \Delta$ be a pencil of degree $d$ curves whose central fiber $Y_{0}=G \cup \Gamma$ is the union of a curve $G$ of degree $d-1$ and a line $\Gamma$ and let $G \cap \Gamma=\left\{p_{1}, p_{2}, \ldots, p_{l}\right\}$ where $l=d-1$. 
Let $X, M, \sigma_{t}, \sigma_{0}, \sigma_{G}, \sigma_{\Gamma}$ and $\mu$ be defined as before. Almost nothing in the argument of Proposition 3.1 needs changing except in the case that $\mathcal{O}(-\mu \Gamma) \otimes M$ is trivial and $\sigma_{G}$ is nowhere vanishing. In this case, following our previous argument, we can show that $\sigma_{\Gamma}$ vanishes at no less than $l-1$ points. The difference is that now we have $l-1=d-2$ and we have to verify that there are only finitely many $\sigma_{\Gamma}$ that vanishes at exactly $l-1$ distinct points. This is more or less obvious because the map from $|L|$ to $\mathbb{P}^{l-1}$ given by (3.4) is dominant and the space

$$
\left\{\sigma_{\Gamma} \mid \sigma_{\Gamma} \text { vanishes at exactly } l-1 \text { distinct points }\right\}
$$

has dimension $l-1$.

Our degeneration method also works for Del Pezzo surfaces.

Theorem 3.1. Let $\widetilde{\mathbb{P}^{2}}$ be the blowup of $\mathbb{P}^{2}$ at $2 \leq r \leq 6$ general points and let $L_{1}, L_{2}, \ldots, L_{k}, \ldots$ be all the smooth rational curves on $\widetilde{\mathbb{P}^{2}}$ with self-intersection -1 . Let $L$ be an ample line bundle on $\widetilde{\mathbb{P}^{2}}$. Then for a sufficiently general curve $D \in|L|$,

1. $\#(D \cap E) \geq \min _{k}\left(D \cdot L_{k}\right)$ for any curve $E \subset \widetilde{\mathbb{P}^{2}}$ that meets $D$ properly;

2. in addition, there exists a set $\Sigma_{D}$ of countably many points on $D$ such that if $\#(D \cap E)=\min _{k}\left(D \cdot L_{k}\right)$ for some $E,(D \cap E) \subset \Sigma_{D}$.

Therefore, for a sufficiently general curve $D \in|L|$ with $\min _{k}\left(D \cdot L_{k}\right) \geq 3$, the complement $\widetilde{\mathbb{P}^{2}} \backslash D$ is algebraic hyperbolic.

Proof. Let $K_{\widetilde{\mathbb{P}} 2}$ be the canonical divisor of $\widetilde{\mathbb{P}^{2}}$. We argue by induction on $\min _{k}(D$. $\left.L_{k}\right)$.

For $\min _{k}\left(D \cdot L_{k}\right)=1$, we need to verify that $g(D) \geq 1$, which is more or less obvious.

Suppose that $\min _{k}\left(D \cdot L_{k}\right) \geq 2$. Let $Y \subset \mathbb{P}^{2} \times \Delta$ be a pencil of curves in $|L|$ whose central fiber $Y_{0}=G \cup \Gamma$ is a union of $G \in\left|L \otimes \mathcal{O}\left(K_{\widetilde{\mathbb{P}}^{2}}\right)\right|$ and $\Gamma \in\left|-K_{\widetilde{\mathbb{P}}^{2}}\right|$ and let $G \cap \Gamma=\left\{p_{1}, p_{2}, \ldots, p_{l}\right\}$.

Let $X, M, \sigma_{t}, \sigma_{0}, \sigma_{G}, \sigma_{\Gamma}$ and $\mu$ be defined as before. Again, the same argument for Proposition 3.1 goes through. We need only to check the following facts, all of which are routine exercises.

1. $l>\min _{k}\left(D \cdot L_{k}\right)$.

2. $H^{0}\left(\widetilde{\mathbb{P}^{2}}, L\right)$ surjects onto $H^{0}(\Gamma, L)$ and

$$
L \otimes \mathcal{O}_{\Gamma}\left(-\sum_{i \neq j} p_{i}\right)=\mathcal{O}_{\Gamma}\left(-K_{\widetilde{\mathbb{P}}^{2}}\right) \otimes \mathcal{O}_{\Gamma}\left(p_{j}\right)
$$

is base point free on $\Gamma$ for each $1 \leq j \leq l$. Hence the map from $|L|$ to $\mathbb{P}^{l-1}$ given by (3.4) is dominant.

3. In the case that $\mathcal{O}(-\mu \Gamma) \otimes M$ is trivial and $\sigma_{G}$ is nowhere vanishing, we can prove that $\sigma_{\Gamma}$ vanishes at no less than $l-1$ distinct points as before. 
But actually, we can do better here since the space

$$
\left\{\sigma_{\Gamma} \mid \sigma_{\Gamma} \text { vanishes at less than } l \text { distinct points }\right\}
$$

has dimension $l-2$ due to the fact that $\Gamma$ is elliptic instead of rational. Therefore, $\sigma_{\Gamma}$ vanishes at no less than $l$ distinct points.

When we go up in dimension, however, some essential difficulties present themselves. For example, in $\mathbb{P}^{3}$, fix a sufficient general surface $S$ of degree $d$ and it is expected that any curve meets $S$ at no less than $d-4$ distinct points [X, Question 2]. Let $Y$ be a pencil of degree $d$ surfaces whose central fiber is a union of a degree $d-1$ surface and a plane and let $X$ be family of curves in $\mathbb{P}^{3}$ meeting $Y$ fiberwise. To carry out the argument as in dimension two, we need to take the limit $X_{t} \cap Y_{t}$ as an element in $A_{0}\left(Y_{t}\right)$, the 0-dimension Chow ring of $Y_{t}$. Of course, we do not know how to do this at present.

\section{Acknowledgements}

I am very grateful to M. Green, who introduced me to the subject of hyperbolic geometry and Kobayashi Conjecture, and to C. Hacon for helpful conversations.

\section{References}

[ACGH] E. Arbarello, M. Cornalba, P. Griffiths, and J. Harris, Geometry of Algebraic Curves, Springer-Verlag, 1984.

[DSW] G. Dethloff, G. Schumacher, and P.M. Wong, Hyperbolicity of the complements of plane algebraic curves, Amer. J. Math. 117 (1995), 573-599. Also preprint alggeom/9312007.

[E-H] D. Eisenbud and J. Harris , Limit linear series: basic theory, Invent. Math. 85 (1986), 337-371.

[H] J. Harris, Moduli of Curves, Graduate Texts in Mathematics 187 (1998), SpringerVerlag.

[X] G. Xu, On the complement of a generic curve in the projective plane, Amer. J. Math. 118 (1996), 611-620.

[Z] O. Zariski, Dimension-theoretic characterization of maximal irreducible algebraic system of plane curves of a given order $n$ and with a given number $d$ of nodes, Amer. J. Math. 104 (1982), 209-226.

Department of Mathematics, South Hall, Room 6607, University of California SANTA BARbara, CA 93106.

E-mail address: xichen@math.ucsb.edu 